\title{
Syndromes and Radiological Manifestations in the Jaws
}

\author{
Viganò Luca ${ }^{1 *}$, Gadalla Khaled ${ }^{2}$, Previderè Greta ${ }^{2}$, Proietto Luca ${ }^{3}$, Pulicari Federica ${ }^{2}$, Rigobello Matteo Maria ${ }^{4}$, Cinzia \\ $\mathrm{Casu}^{5}$
}

${ }^{1}$ Department of Radiology, ASST Santi Paolo e Carlo, San Paolo Dental Building, University of Milan, Italy

2Dentistry student, IRCCS Ca' Granda Foundation General Hospital, University of Milan, Italy

${ }^{3}$ Dentistry student, ASST Santi Paolo e Carlo, San Paolo Dental Building, University of Milan, Italy

${ }^{4}$ Dentistry student, IRCCS Istituto Ortopedico Galeazzi, University of Milan, Italy

${ }^{5}$ Department of Surgical Science, OBL Oral Biotechnology Laboratory, University of Cagliari, 09124 Cagliari, Italy

DOI: $10.36347 /$ sjds.2020.v07i11.003

| Received: 02.11.2020 | Accepted: 11.11.2020 | Published: 16.11.2020

*Corresponding author: Viganò Luca

Abstract

Radiological investigations are fundamental in the diagnostic processes of bone lesions. In the pathologies of the jaws, this type of investigation can be useful in predicting a systemic pathology and anticipating its clinical diagnosis, so it is possible to intervene on the manifestations with an adequate treatment plan for the patients, improving their quality of life.

Keywords: Syndromes Radiological Jaws.

Copyright $\odot 2020$ The Author(s): This is an open-access article distributed under the terms of the Creative Commons Attribution 4.0 International License (CC BY-NC 4.0) which permits unrestricted use, distribution, and reproduction in any medium for non-commercial use provided the original author and source are credited.

\section{INTRODUCTION}

The aim of this study is the analysis of different syndromes that induce bone changes in the maxillofacial region. The objective is to intercept or diagnose syndromic diseases through radio-diagnostic investigations, allowing the development of an appropriate therapeutic plan through a multidisciplinary approach and where possible anticipate its clinical course.

In some syndromes, radiological examination can be fundamental in order to anticipate its clinical manifestations. Furthermore, it can induce the clinician to intervene early avoiding that an initially benign and/or asymptomatic pathology (e.g. adenomatous polyps in Gardner's Syndrome) turns into a malignant tumor [1]. The early setting of paraneoplastic syndromes and tumor pathologies, in particular, can be of fundamental importance for the prognosis of the patient to be treated [2].

The knowledge of the district anatomy and how the structures are physiologically radiological are fundamental. This allows identifying and recognizing the lesions of the maxillofacial district, remembering that the standard top-level radiographic image, orthopantomography, is the two-dimensional image of an anatomical structure and that it could present enlargements, distortions or overlaps that could lead to error.

Anatomical structures, radiologically, according to bone density are presented as radiopaque or radiolucent. Within the two extremes there will be bone densities highlighted by a gray scale, which can allow identifying any focal lesions.

The jaw lesions can be classified also based on the etiopathogenesis in odontogenic or nonodontogenic. However, a significant number of pathologies, based on the radiological examination, can be classified as mixed or initially radiolucent and subsequently become radiopaque [3].

The diagnostic investigation, in dentistry, is mainly based on: anamnesis, objective clinical examination and orthopantomography. Based on the outcome, the radiological examination, in the presence of bone alterations, will be able to decide whether to perform a second level examination such as CT or Cone Beam Computer Tomography (CBCT). To these two imaging methods it can also be useful to add a histopathological examination of the present lesion [4].

\section{MATERIALS AND METHODS}

This research is an overview of some studies on the manifestations of certain syndromes that can be detected radiologically at the level of the orofacial district. 
Both pathologies strictly related to the oral cavity and systemic pathologies presenting manifestations at the level of the maxillofacial region have been evaluated, in order to provide a quick support to the dentist for their early classification.

\section{Below is the list of pathologies treated:}

- Ghost cell odontogenic tumors (closely related to the oral cavity)

- Paget's disease, renal osteodystrophy, Gardner's syndrome, ectodermal dysplasia, Langherans cell disease and Crouzon's disease. (systemic alterations with maxillofacial manifestations)

The articles considered in this review were selected from a search conducted in the PubMed database. This article contains images, taken from other selected articles that explain the focal alterations on a maxillofacial level.

\section{DISCUSSION}

\section{Ghost cells odontogenic tumors}

Odontogenic tumors originate from one or more dental tissues and their morphogenesis reflects the process of odontogenesis[5].

We will specifically evaluate odontogenic tumors characterized by "ghost cells", that they are ellipsoidal keratinized epithelial cells characterized by loss of nucleus, conservation of cellular contours and calcification potential.

The classification of these tumors has been controversial and debated over the years. In 2005, the WHO ended the discussion by classifying as ghost cell odontogenic tumors: cystic calcifying odontogenic tumor (CCOT), dentinogenic ghost cells tumor (DGCT) and the malignant counterpart, ghost cells odontogenic carcinoma (GCOC)[6].

WHO defined the DGCT as "a locally invasive neoplasm, characterized by islands of epithelial cells similar to ameloblastoma, immersed in a mature stroma of connective tissue? Aberrant keratinization can be found in the form of ghost cells in association with varying amounts of dysplastic dentin [7].

There are two types of DGCT: the extraosseous (peripheral) type and the intraosseous (central) type. [8]. Intraosseous DGCTs are more aggressive, tend to be invasive and the age group of patients varies from 12 to 75 years with a prevalence towards the fourth decade $[9,10]$.

The extraosseous type has limited growth potential and occurs more frequently around the sixth decade [11]. The lesions are more common in males than in females, equally involving the maxilla and the mandible [12].

Intraosseous lesions vary in size from 1 to more than $10 \mathrm{~cm}$ in diameter. They occur mainly in the canine region up to the first premolar and the clinical features include visible swelling, the facial asymmetry due to the expansion of the jaw and / or obliteration of the maxillary sinus and relative infiltration of soft tissues is evident. Swelling can be associated with a painful occasionally accompanied by secretion of pus and mobility or displacement of the affected teeth $[6$, 8].

Extraosseous lesions show predilection for the anterior regions and usually arise in the edentulous areas. They appear as solid and painless nodules on the gingival or alveolar mucosa [8].

Radiologically, DGCTs present themselves as radiolucent lesions or mixed lesions depending on the degree of calcification. In most cases the lesions are unilocular but multilocular lesions can also be observed. These tumors are generally well defined, often expansive and can cause resorption and dislocation of the roots of adjacent teeth [13] (Fig.1) Occlusal radiographs show bicortical expansion [14].

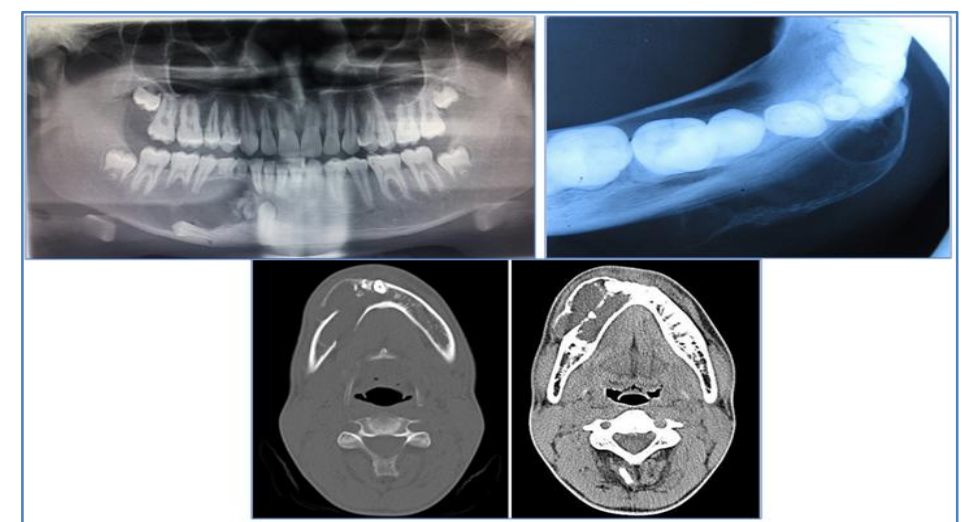

Fig-1: Courtesy of Dr. Yash Agrawal: Radiographic images showing the radiopaque and radiolucent lesions characteristic of DGCTs. Above left, panoramic radiograph showing a predominantly radiolucent lesion with visible opacification areas extending from the right mandibular later incisor to the second molar. Above right, radiolucent lesion with radiopaque calcium patches with thinning and expansion of the vestibular and lingual cortex. Below, computer tomography (CT) which shows the presence of multiple compartments mainly in the buccal cortex with thinning of the lingual cortex 
Histologically, the tumor is uncapsulated, predominantly solid and infiltrating. The main components of the tumor include epithelial cells and ghost cells similar to ameloblastoma, often accompanied by dentin dysplasia. Epithelial cells with ameloblastic differentiation are arranged in islands and nests within mature fibrous connective tissue. Scattered between the tumor epithelium are variable quantities of ghost cells [8] (Fig.2).

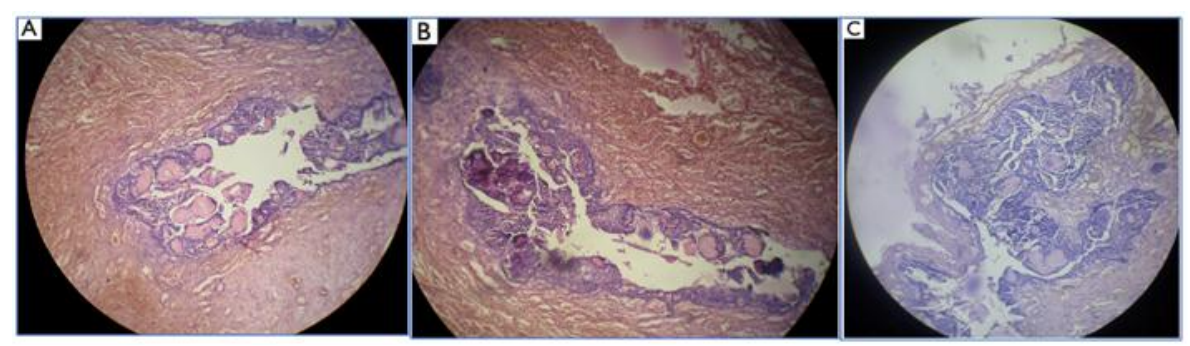

Fig-2: Courtesy of Dr. Smita Bussari: (A) Photomicrograph of lesion showing rounded sheets or odontogenic epithelial lining with phantom cell foci $(\mathrm{H \& E}, 10 \times)$; (B) Areas of dystrophic calcification within the lesion along with ghost cell seen. Epithelial islands seen within connective tissue $(H \& E$ 10x); (C) Invagination of the epithelium within the connective tissue (H\&E 10x).

Ghost cells are described as ellipsoidal keratinized epithelial cells characterized by loss of nucleus, conservation of cellular contours and calcification potential (Fig.3).

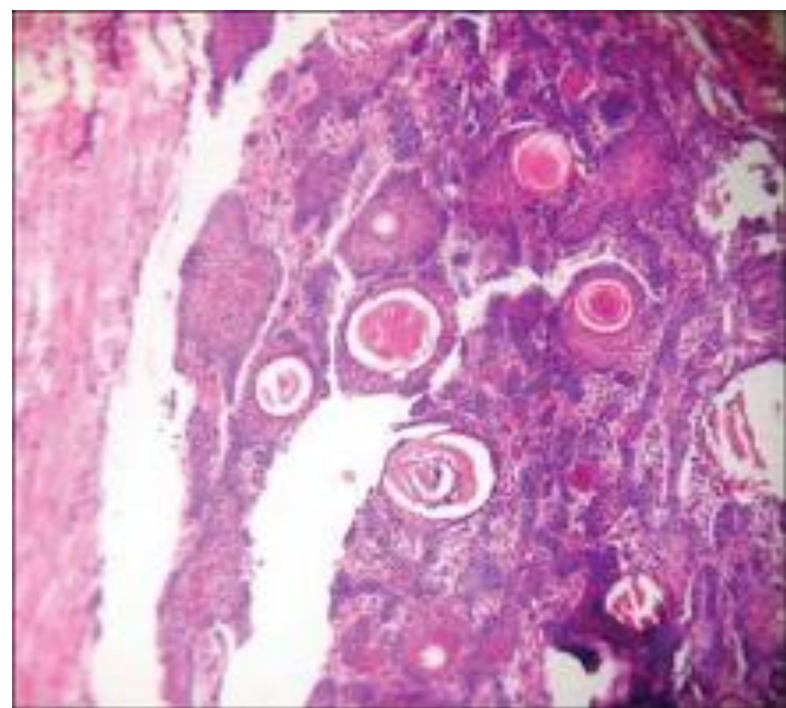

Fig-3: Courtesy of Dr. Smita Bussari: Histological detail of the ghost cells

The motivation for their formation is unknown but there are various theories such as:

- Transformation of epithelial cells,

- The metaplastic transformation of the odontogenic epithelium and the squamous metaplasia with secondary calcification due to ischemia,

- The degeneration of epithelial cells or secondary to an involutionary process of apoptosis.

It should be specified that the presence of ghost cells is not pathognomic of the DGCT as they can also be identified in other neoplasms with which the DGCT enters differential diagnosis and described later [6].

The other histopathological feature of the DGCT is the formation of dysplastic or dentinoid dentin in which ghost cells can become trapped. It appears as an amorphous mass of eosinophilic material.

Also in this case the formation of dysplastic dentin is unknown but various hypotheses have been made by various authors in the literature. Gorlin et al. [15] believed that it represented an inflammatory response to the ghost cell masses.

Abrams and Howell [6] have stated that the masses of "ghost cells" cause the granulation tissue to lay down the juxtraepithelial osteoid which can calcify becoming dentinoid.

For Singhaniya et al. [16], the dentinoid represents a metaplastic change in connective tissue without the participation of granulation tissue.

The etiology of this lesion is still unknown but it has been suggested that it is based on a mutation of the $\beta$-catenin. It is a co-activator of the lymphoid enhancement factor (Lef), which is a specific transcription factor that mediates Wnt signaling during odontogenesis. So a mutation at the Wnt/ $\beta$-catenin level can cause a change during tooth development [17].

The DGCT goes in differential diagnosis with the adenomatoid odontogenic tumor (AOT) and the calcifying epithelial odontogenic tumor (CEOT).

AOT is an odontogenic tumor associated with missing teeth and present with painless swelling of the jaw. Radiographically, it appears radiolucent, unilocular, well circumscribed and with poorly defined radiopacity groups. These are better visualized on intraoral periapical radiographs than on panoramic radiographs. But in AOT the displacement of neighboring teeth is usually observed due to tumor expansion rather than root resorption typical of DGCTs [18]. 
CEOT is a benign odontogenic tumor that is often associated with painless swelling and slow growth in the molar and premolar regions. Radiographically, depending on the development phases, it can present variable, unilocular or multilocular radiographic aspects. X-rays taken in the early stage of these tumors can reveal a completely radiolucent area around the crown of the tooth. Then, multiple radiopacities of varying sizes may develop within the radiolucent area. In some cases, small, thin and opaque trabeculae can be found giving a multilocular or honeycomb model different from the appearance of the DGCT [19].

CCOT is also related to DGCT, with both lesions presenting epithelium similar to ameloblastoma, ghost and dentinoid cells. However, CCOT is primarily cystic, while DGCT is primarily solid [8].

Finally, it is important to distinguish the DGCT from its malignant counterpart, the ghost cell odontogenic carcinoma (GCOC).

GCOC is a rare malignancy that can originate primitively or more frequently from the malignant change in the DGCT.

Both lesions can present ghost cells and infiltrative growth. However, the overproliferation of small cells with poor cytoplasm, hyperchromatic nucleus, and lively mitotic activity and in some cases necrosis, help to distinguish GCOC from DGCT.

The levels of ki-67 and p53 are also fundamental for the distinction. Both are overexpressed in the GCOC, while they are poorly expressed in the DGCT [20].

Early diagnosis of the DGCT is essential for a better patient prognosis and the treatment plan usually differs for both variants due to the difference in recurrence frequency and malignant potential.

\section{Paget's disease}

Paget's disease (PBD) is a chronic metabolic disorder characterized by areas with increased bone remodeling processes, which lead to rapid bone resorption and disorganized bone formation. The regions of the skeleton involved can be unique (monostotic) or more frequently multiple (polyostotic).

It generally occurs around the fourth-fifth decade and involves the pelvis, the spine, the femur and the tibia more frequently. Rarely it can also involve the jaws, with a maxilla/mandible ratio of 2:1[21].

The manifestations of the disease, on a skeletal level, have numerous inversion lines that give the lamellar bone a characteristic mosaic appearance. There is abundant local vascularization and fibrous tissue in the marrow. Clinical symptoms include pain; bone deformity and can induce fracture of the affected bone, although the initial course of the disease may be asymptomatic [22].

The complications of Paget's disease are related to the stages of osteolytic and sclerotic alterations. They include nerve compression syndrome, hearing loss when it affects the petrous bone, pathological fractures and spinal stenosis [23]. The different areas of osteolysis, characteristic of the pathology, become evident on the radiographic investigation.

The diagnosis of PBD is often fortuitous, since there are no specific signs indicative of the pathology: it is frequently formulated due to changes found on the radiographic level or bone changes. In the specific case of a dentist, the most frequent cases of early diagnosis of Paget's disease present dental malpositions, prognathism of the jaw and alterations found through radiographic images.

Furthermore, the diagnosis can also be made in the presence of high levels of alkaline phosphatase in an apparently healthy individual. In addition, genetic tests can be useful, especially in identifying specific regions of chromosomes 5 and 6 present in the disease, such as the RANK and SQSTM1 genes [21].

By performing a radiographic status, areas of radiolucency and radiopacity in the periapical portion of the roots of various dental elements can be detected [21] (fig.4).

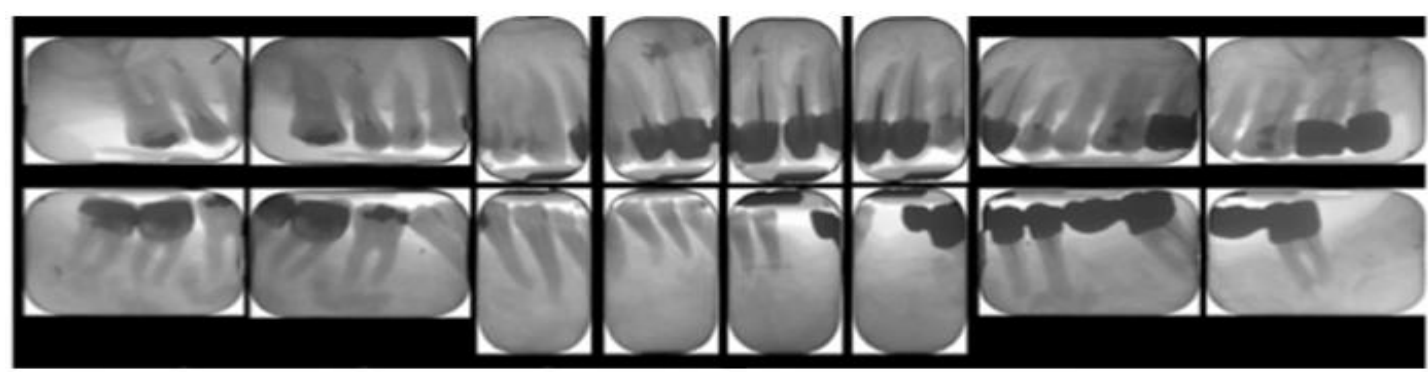

Fig-4: Courtesy of Dr. Marco Cabras: Radiographic status with areas of radiolucency and radiopacity in the periapical portion of the roots of 4.6, 4.7, 4.8 and 3.5 . 
Thanks

to

orthopantomography,

hypercementosis areas with dislocation of the teeth involved, cortical thickening of the jaw body and mixed areas of radiolucency and radiopacity on both sides of the jaw become evident[22] (fig.5)

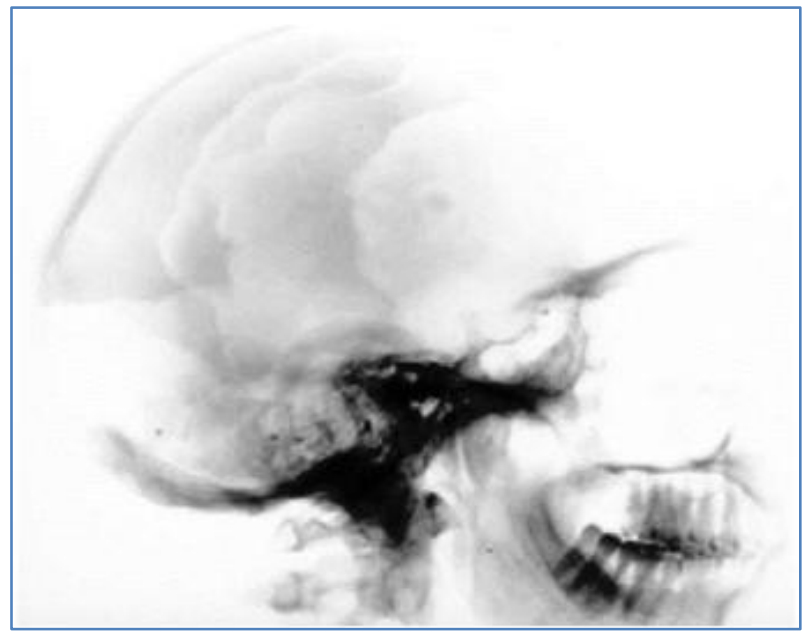

Fig-5: Courtesy of Dr. Mayank Patel: Lateral X-ray of the skull showing numerous multiple levels of osteolytic changes in a patient with PD in Jarrah Ali Al-Tubaikh, Maximilian F. Reiser, "Congenital Diseases and Syndromes, An Illustrated Radiological Guide," SpringerVerlag Berlin Heidelberg, 2009

In addition, through the execution of a $\mathrm{CT}$ we can notice areas of bone deformation with areas of thickening alternating with areas of bone rarefaction of the jaws [21].

On intra-oral examination, increased dimensions and altered shape of the mandibular alveolar crest and pseudo micrognathia of the maxillary arch can be found [22].

Often the disease can also cause dislocation of the dental elements with consequent occlusal changes. (fig.6)

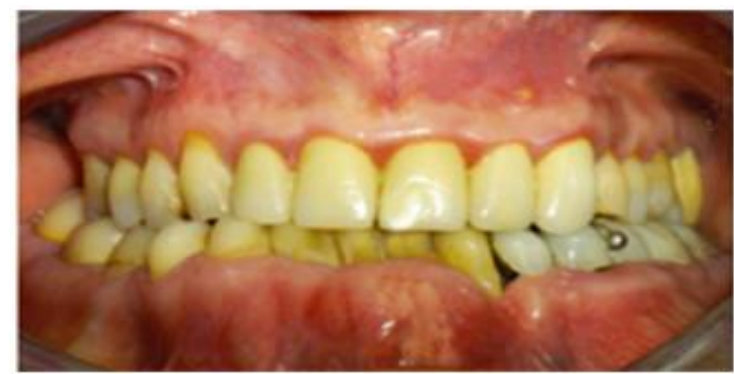

Fig-6: Courtesy of Dr. Marco Cabras: Intra-oral view showing the slippage of the lower incisors and canines

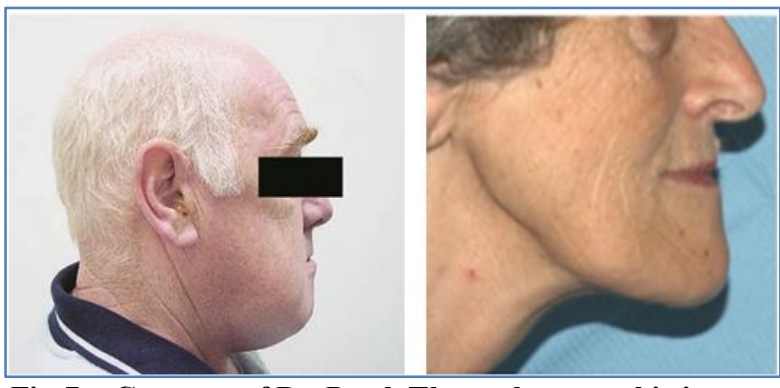

Fig-7a: Courtesy of Dr. Patel. Three photographic images showing the prognathism of the jaw in different patients. Figure 7b courtesy of Dr. Marco Cabras

With the extra-oral examination, we realize that the facial profiles of the patients are significantly altered, since in most cases they have prognathism of the jaw (fig.7 a-b).

Along with radiographic investigations, incisional biopsies are also used, which show greater osteoblastic activity and irregular cortical bones with the presence of a hematophilic inversion line which gives the characteristic mosaic motif; moreover, the medullary spaces are filled with fibro-vascular connective tissue [22].

Many patients experience an abnormal increase in alkaline phosphatase and treatment with bisphosphonates normalizes the serum alkaline phosphatase level [24].

Bisphosphonates are administered for the treatment of PD because they effectively restore mineral density and bone structure, reducing the incidence of fractures. Their administration can induce maxillary osteonecrosis. This type of drug inhibits the function, activation and survival of osteoclasts. It is possible to find examples where implant treatment in PD patients has been successful, even in unfavorable conditions of bone density, such as those considered "poor" or with standard results of "cotton balls"[25].

Early diagnosis of PD by dentistry and timely treatment allow limiting hypertrophy and, in some cases, evening mandibular pain [24].

\section{Renal osteodystrophy}

Renal osteodystrophy (ROD) refers to a spectrum of bone diseases caused by pathological changes in the metabolism of calcium, phosphate and bone. These changes are evident in the context of endstage chronic kidney disease and secondary hyperparathyroidism. The various mechanisms by which it occurs are: tubular acidosis with consequent dissolution of hydroxyapatite and demineralization of the matrix; reduction of phosphate secretion, chronic hyperphosphataemia and hypocalcaemia; decrease in vitamin D resulting in hypocalcaemia [26]. 
This pathology frequently affects the orofacial region and presents characteristic radiological manifestations [27]. It also shares clinical, histological and radiological similarities with various benign fibroosseous conditions affecting the craniofacial region and its recognition is fundamental for prevention, choice of therapy and prognosis.

Renal osteodystrophy can present large orofacial and dental manifestations, such as gingival hyperplasia, periodontal disease, xerostomia, lichen planus, uremic stomatitis, candidiasis, herpes simplex, delayed dental eruption, enamel hypoplasia, dental mobility but also a loss of density at the level bone [28]

Radiologically, the "frosted glass"; characteristic diffused throughout the mandibular or maxillary portion is revealed, useful for recognizing the disease but must be differentiated from two other pathologies: from the fibrous dysplasia that is usually confined to a single quadrant and does not influence widely maxilla and mandible; and Paget's disease also associated with other dental and systemic manifestations[29].

On intra-oral examination, mandibular swellings and/or swelling of the palate can be found. A characteristic of the disease is also an excessive growth and deformity of the mandibular bone which can be single or bilateral [29].

One of the first symptoms may be an unpleasant uremic odor in the mouth, especially in the morning. This uremic stench is due to a high concentration of urea in the saliva. Xerostomia is a common finding in these patients, as enamel hypoplasia may develop due to disorders of calcium and phosphate metabolism. A compromised balance of calcium and phosphate can cause a narrowing of the pulp chamber [30].

There are also other systemic features such as a typical arched form of the lower limbs, short stature and slow growth due to the alterations of calcium, phosphates and vitamin D underlying the pathology [28].

In patients with renal osteodystrophy there is an increase in osteoclastic resorption of bone trabeculae and a greater proliferation of osteoblasts present on the bone surface. In addition to these altered components, an accumulation of fibrous tissue is also observed. Multinuclear giant cells may also be present [29].

Together with the radiographic results, biochemical markers are used in the diagnosis and monitoring of renal osteodystrophy. Serum parathyroid hormone (PTH) and total alkaline phosphatase (ALP) are the most used biochemical tests. To these is also added a control of the patient's diet by changing the intake of calcium and phosphates and trying to restore the balance altered by the pathology [30].

\section{Gardner's syndrome}

Gardner's syndrome (GS) is a rare autosomal dominant disorder characterized by the presence of polyposis of the colon with a remarkable tendency towards neoplastic transformation (adenocarcinomas), multiple osteomas, skin and soft tissues tumors. This syndrome constitutes a variant of the most common familial adenomatous polyposis (FAP) and can occur at any age (from 2 months to 70 years old) [31, 32].

The main mutation associated with GS is located in the 5q21-q22 locus of the colon adenomatous polyposis gene (APC). Family predisposition is a determining factor for the majority of GS patients, while in about $25-30 \%$ of cases this mutation occurs spontaneously. More than 1400 different mutations of this gene have been discovered and in relation to the specific mutation of the APC gene, different levels of severity of the GS can be determined (number, time of onset and malignant potential of the adenomatous polyps); each one of them is associated with peculiar extraintestinal pathological manifestations [33].

Intestinal polyposis is also a pathology that, if left untreated, has a $100 \%$ chance of malignant transformation around the fourth decade of life [32]. Therefore, it is of fundamental importance for the dentist to know and diagnose oro-maxillofacial anomalies associated with GS. These anomalies may be the earliest to manifest and usually precede adenomatous polyposis by at least 10 years.

The main pathognomonic sign related to GS is the presence of multiple osteomas associated with the maxillofacial district. Osteomas can be found in 65$80 \%$ of patients with GS [33]. Osteomas are benign bone tumors and are generally diagnosed in the second decade of life. According to Ida et al., the detection of three or more osteomas can be a valid screening criterion for GS [34]. Radiologically these neoplasms appear as well defined radiopaque masses, with a density similar to that of normal bone.

Furthermore, osteomas are more frequently located near the maxilla (especially at the level of the angle and lower surface of the jaw) and the skull. They are divided into peripheral (periostal) and central (endostal) osteomas [35, 36]. Unlike endostal osteomas, which often are asyptomatic and are detectable with radiodiagnostic investigations, periosteal ones manifest themselves clinically as prominent masses which, while growing, cause facial deformity and functional and aesthetic problems. CT is considered the best imaging method for diagnosing osteomas (fig. 8-9). 


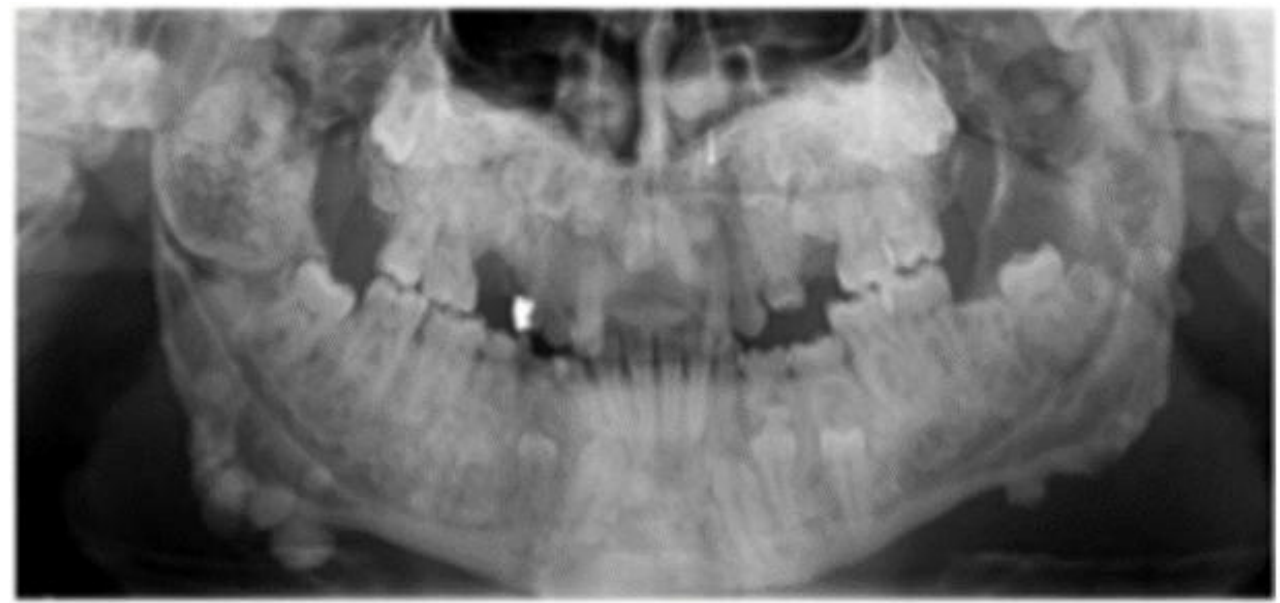

Fig-8: Courtesy of Dr. Burak: Panoramic view of a 21-years old patient presenting multiple osteomas. A particulary large lobulated osteoma is present in the right condyle and coronoid process that impacted both permanent and deciduous teeth
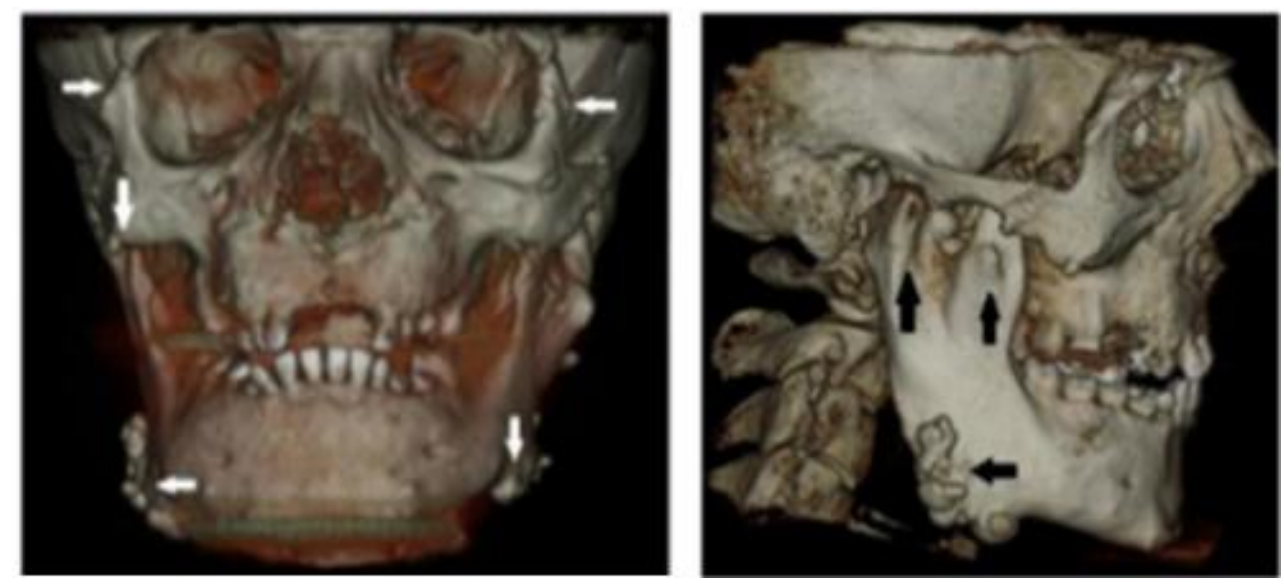

Fig-9: Courtesy of Dr. Burak: Dental volumetric tomographic (DVT) images showing multiple osteomas (white and black arrows)

Dental anomalies are found in $30-75 \%$ of patients with GS [33] and mainly include congenital agenesis, hypodontics, hypercementosis, odontomas [37], supernumerary teeth [38] impacted teeth, fused or unusually long roots and odontogenic cysts [39].

Odontogenic myxoma is present among odontogenic tumors associated with GS [40]. Odontogenic myxoma is a rare benign neoplasm, locally invasive and represents $1.5 \%$ of all tumors of the maxillary bones. Radiographically, this tumor can appear as a well-circumscribed radiolucency area, in which there are numerous multilocular compartments that give it a multi-lobed image. Furthermore, unilocular radiolucent areas can be observed in $35 \%$ of cases. The diagnostic certainty of this neoplasm is given only by histological examination [41].

Epidermoid cysts [42] represent the main skin lesions related to GS and can be found in about $50-65 \%$ of patients. Epidermoid cysts can develop on the back, head and neck; they have no malignant potential and generally arise during puberty $[37,43]$.
Additional extraintestinal manifestations related to GS may be: desmoid tumors [32], congenital retinal pigment ephitelial hypertrophy (CHRPE) [44, 45] soft tissue lesions (fibroids, neurofibromas, keloids, leiomyomas, lipomas) and tumors not localized in the colon (thyroid, liver, bile ducts, and central nervous system).

GS is in differential diagnosis with other diseases associated with family polyposis such as Peutz-Jeghers syndrome, Lynch syndrome, familial or hyperplastic juvenile polyposis and hereditary mixed polyposis syndrome. If possibile, Clinical diagnosis is confirmed by genetic tests. If the APC gene mutation is identified in a family member, the genetic test should also be extended to first degree blood relatives.

\section{Ectodermal dysplasia}

Hypohidrotic ectodermal dysplasia (HED) is a genetic disorder of ectodermal development with malformations of the resulting structures. 
Viganò Luca et al., Sch J Dent Sci, Nov, 2020; 7(11): 183-195

It includes 3 subtypes, not clinically differentiable, which present, as a cardinal sign, a sweating disorder:

- Christ-Siemens-Touraine syndrome (CST),

- Autosomal recessive HED (AR)

- Autosomal dominant HED (AD)

A fourth rare subtype is identified as HED with immunodeficiency. The AD and AR forms affect both genders equally. In the X-linked form, female's carriers may be asymptomatic or have a milder phenotype.

HED is caused by genetic mutations of the ectodisplasin / NF- $\mathrm{BB}$ pathway, necessary for the correct development of ectodermal structures.

CST syndrome is due to mutations of EDA (Xq12-q13.1), which encodes the ectodysplasin-A epithelial morphogen of the family of tumor necrosis factors. The AR and AD HEDs are due to mutations of EDAR (2q13), which encodes the ectodysplasin-A receptor, or EDARADD, which encodes the EDARassociated death domain protein (EDARADD). Immunodeficiency HED is due to IKBKG mutations (Xq28). In some cases of HED, mutations in WNT10A, TRAF6, NFKBIA or EDA2R have been identified.

Phenotypically, the pathological patient present sparse hair, absence of body hair, deficiency of sweat glands. They often have a typical facies, with prominent forehead, saddle nose, prominent lips, periorbital wrinkles and pigmentation. Sometimes a decrease in subcutaneous fat, muciparous glands in the respiratory and gastrointestinal tract can be found. Growth retard may be present.

Oral cavity can be characterized by anodonzia or oligodonzia [46]. Dental anomalies are found in both deciduous and permanent teeth, sometimes presenting conical incisors and canines [47]. Agenesia, affect frequently the upper lateral incisors, second molars and premolars and mandibular incisors [48].

The diagnosis is often set off after episodes of hyperthermia or delayed tooth eruption. The absence of the sweat glands can be highlighted by skin biopsy and, in a non-invasive way, by confocal microscopy or by taking fingerprints of the hands or feet with graphite. The function of the sweat glands is assessed with the quantification of sweating induced by pilocarpine. The diagnosis is confirmed by genetic tests.

From a radiological point of view, the execution of orthopantomography and computerized tomography, which can highlight the defects affecting the dental elements that must cause a systemic pathology such as HED to be differential diagnosed, is confirmed as useful.
In this pathology, the absence of teeth in the jaw, the abnormal formation of the crown of the maxillary incisors, malformation of the root of the molars and taurodontism are more evident in males. On the other hand, cases of hypodontics are observed in heterozygous females. As regards the dimensions of the crowns of the dental elements, these appear to be of reduced dimensions in the mesio-distal and buccolingual sense. The most common teeth are the first molars, the upper and canine upper and lower incisors [48].

There is a loss of bone tissue especially at the edentulous saddles, which predisposes patients suffering from ectodermal dysplasia to periodontal disease.

Anodonzia can be seen in both males and females, generally presenting 10 or less elements. In many cases, this condition, associated with a reduction in the vertical size of the alveolar bone, leads to maxillary hypotrophy and mandibular hypertrophy [48].

It is possible to say that hypohidrotic ectodermal dysplasia has a particular relevance for dentistry and requires a multidisciplinary approach to trace a correct therapy for the restoration of masticatory function, growth and oral and dental health, also using imaging methods. The use of diagnostic investigations allows an early diagnosis and indirectly improve the quality of life of patients affected by the disease.

\section{Langherans cell histiocytosis}

Langherans cell histiocytosis (LCH) is pathology of unknown etiology, characterized by the pathological proliferation of Langherans cells in different areas. It is a disease that mainly affects the population in childhood, but it could be also observed in adulthood.

The head and neck are the most affected districts, with a prevalence of $50 \%$ of cases of lesions in the skull and $5-10 \%$ lesions in the mandibular and maxillary bones, but it is possible to find lesions in different tissues, such as skin, hypothalamus, liver, lung and lymphoid tissue [49]

Cancer cells derive from dendritic cells that express markers such as HLA-DR, S-100 and CD1a. Using electron microscopy it is possible to observe Birbeck granules, typical rod-shaped structures found in the cytoplasm. The typical concentration of Langherans cells in the skin and bone is due to the fact that the neoplastic cells show an altered expression of the receptors for chemokines, which allows them to migrate to the skin and bone marrow and lymphoid organs.

Clinically we can distinguish two forms: multifocal and multisystem Langherans cell histiocytosis (Letterer-Siwe disease) that affects 
children under two years of age, showing a characteristic eruptive skin lesion as a consequence of infiltration of Langherans cells. Lymphadenopathies, extensive osteolytic lesions, lung infiltrates and hepatosplenomegaly are also observed in affected subjects. The second form, limited to single organs or skeletal segments, is called monosystemic monofocal and multifocal Langherans cell histiocytosis (known as eosinophilic granuloma).

In some cases multiple bone lesions in children or young adults, develop a particular triad caused by cranial osteolytic lesions, exophthalmos and diabetes insipidus.

In the oral cavity of children affected by the pathology in its multifocal and multisystemic sense,we can observe mucous ulcerations and destruction of the jaw bones, dental mobility and alveolar bone destruction similar to that observed in periodontal disease.

In the eosinophilic granuloma form, the lesions preferentially affect the cranial bones, the jaw and the maxillary, with single or multiple lesions, without visceral involvement.

The rarity of the disease and its multifaceted manifestations makes a difficult diagnosys and recognition, it is therefore necessary that it has been taken into consideration among the possible hypotheses.

Pediatricians or dentists could suspect it in children, especially if there is bone lesions discovered accidentally or there is unexplained pain or fractures, dental problems or body deformations.

The hypothesis can be supported by the swollen lymph nodes in the neck and the presence of characteristic spots on the skin.

Adults, on the other hand, come to the attention of the doctor more often for breathing problems caused by the presence of granulomas in the lungs.

In both cases, the biopsy, which could be performed in the skin, bone or mucous membrane of the intestine, could be useful to settle any doubt.
LCH patients often initially exhibit maxillary symptoms and the exact differential diagnosis of $\mathrm{LCH}$ in the orofacial district is essential for planning early treatment of the disease.

X-rays of LCH lesions are often difficult to understand because the disease can mimic a wide variety of pathological conditions, such as periapical cysts, tumors of odontogenic and non-odontogenic origin, osteomyelitis, vascular malformations and malignant neoplasms. Despite this, multiplanar radiological investigation methods such as computed tomography, magnetic resonance imaging are widely used for the diagnosis of head and neck injuries, while they are rarely used in the diagnosis of lesions located in the maxilla, where traditional radiography is used [50].

The characteristic lesions present in the jaws, visible on radiographic examination, appear as radiolucent, destructive, and often well-defined with irregular margins. The size is variable, occasionally the loss of bone tissue becomes serious enough to erode the cortical bone and the corrispondent teeth appear "floating in the air [51]". Bone lesions therefore appear as non-calcified with lytic area free of peripheral sclerosis [48]. The first symptoms of histiocytosis can initially occur in the oral cavity, prompting the patient to present himself to the dentist for "continuous gingivitis" or "dental abscesses" up to necrotic or ulcerative lesions or painful edema at the jaw level.

The massive loss of bone tissue is characteristic of these lesions, sometimes it is also possible to find cystic lesions at the alveolar bone level, with poor prognosis for the dental elements involved, which must be extracted for high mobility. Differential diagnosis in these cases must include cysts and odontogenic tumors, primary bone tumors, osteomyelitis, metastasis, multiple myeloma and giant cell granuloma [49].

In the skull, the radiographic images show lytic lesions with a holey pattern, without evidence of periosteal reaction or marginal sclerosis, known as a "geographical map skull". In some cases it is possible to observe a central mass of residual bone inside the lytic lesion, called the "bull's eye" aspect. These lesions may indicate the presence of the eosinophilic granuloma (fig.10) 


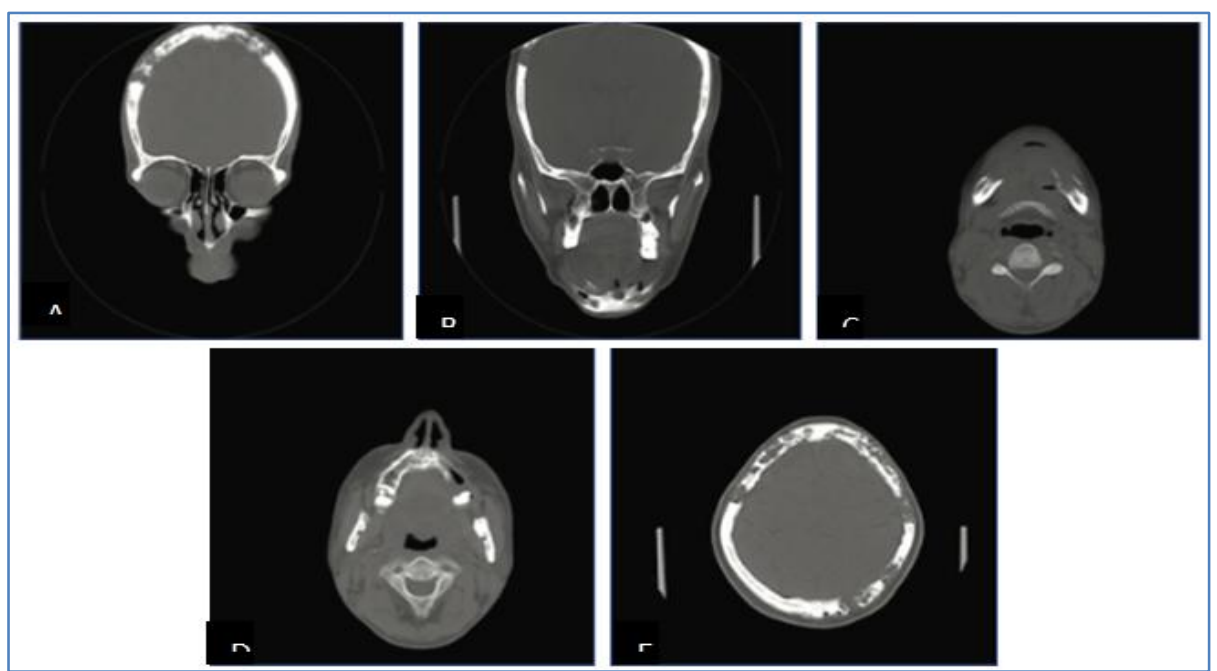

Fig-10: Courtesy of Dr. Kyung-Hoe Hu: (A) Coronal CT sections showing multiple osteolytic lesions of the skull. (B) Pseudo multilocular aspect observed in the anterior mandibular region (black arrows). (C) axial section of the mandible showing a periosteal reaction involving the lingual cortex of the left posterior mandibular region (black arrow). (D) Obvious destruction of the left maxillary alveolus. (E) Appearance of the loculated skull (from left to right)

Through CT and MRI it is possible to observe a mass of soft tissue that goes with the lesions, surrounding them with alteration of the bone marrow. It is also possible to use the scintigraphy to evaluate the extent of the lesion and monitor its course. (fig.11)

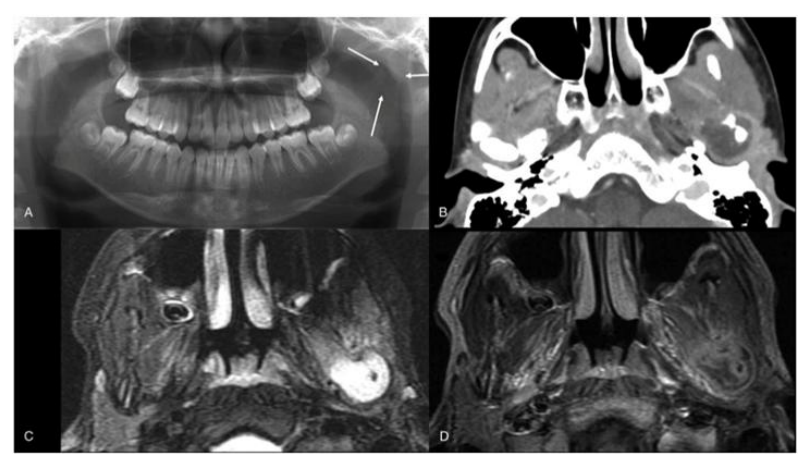

Fig-11: Courtesy of Dr.Jayam Raviraj: Langerhans cell histiocytosis in the left condyle in an 11-year-old boy.

Panoramic radiography (A), enhanced computed tomography with axial control (B), MRI (C - D). Note the intensity of the fluid inside the lesion

In conclusion, the evaluation by radiographic imaging can be predictive of a systemic pathology such as Langherans cell histiocytosis, which must be placed in differential diagnosis by the dentist among the pathologies affecting the jaw bones, in order to provide a diagnosis timely and plan promptly a treatment of the injury.

\section{Crouzon syndrome}

Crouzon syndrome is a genetic condition characterized by skeletal and facial deformities resulting from premature fusion of coronal structures (craniosynostosis)[52] of two or more sutures. It is a dominant full-penetrating autosomal genetic syndrome caused by a mutation in the Fibroblast Growth Factors Receptor 2-3 (FGFR) on chromosome 10[53].

The gene for coding FGFR-2 (transmembrane protein) is located on 10q26. FGFR-2 contains three extracellular immunoglobulin domains (IgI, IgII, IgIII), a single transmembrane domain, and a divided domain of tyrosine kinase (TK1 and TK2). The FGFR-2 protein increases tyrosine phosphorylation of several intracellular proteins by binding to FGF and even autophosphorylation. Most of the pathogenic variants related to CS are located in the IgII domain. The FGFR2 receptor is also responsible for the differentiation of odontoblasts during development and "nonsense" mutations would lead to acceleration in differentiation. Pathological variants of this protein are implicated in Pfeiffer and Apert syndromes [54].

Deformities can occur at both intra-oral and extra-oral (systemic) levels. At the extra-oral level we observe brachycephaly, hypertelorism, proptosis and a beaked nose [52], while at intra-oral level it can manifest with jaw prognathism, presence of supernumerary or missing elements and jaw arch "V"[55]. The absence of elements is more common than the presence of supernumeraries [56]. This syndrome also affects the eruption of teeth causing a delay (fig.12). 

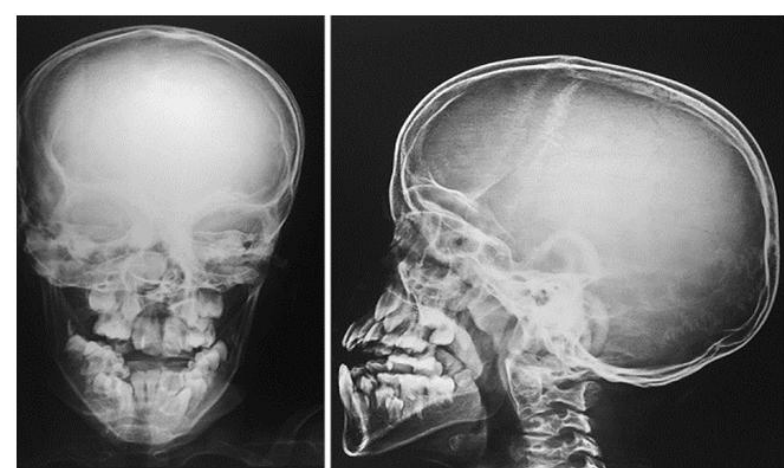

Fig-12: Courtesy of Dr. Anupriya Kaushik: Crouzon disease. Postero-anterior view (PA) and teleradiography of the skull showing hypoplastic jaw, mandibular prognathism and brain impressions

Crouzon syndrome ends up in differential diagnosis with other craniosynostosis-related pathologies such as Pfeiffer syndrome, Apert syndrome, Saethre-Chotzen syndrome, Carpenter syndrome, and Jackson-Weiss syndrome [57].

The coronal and sagittal sutures begin to close around the first year of life, the growth of the skull also depends on the activity of the sutures, and premature ossification can limit its development.

To diagnose Crouzon syndrome, it is very important to evaluate the family history, the risk of a gene mutation is higher if there is a case within the patient's family; clinical aspects are assessed and then person's symptoms. If the clinician has not ruled out the syndrome from diagnosis, then it may require a genetic test to evaluate any mutations. Examinations such as MRI (magnetic resonance imaging), CT or orthopantomography (after 5 years) can also be performed. These imaging techniques are used to detect non-somatic alterations such as increased intracranial pressure and allow for better prognostic diagnosis in children.

The most frequently reported symptoms are hearing loss, Meniere's disease, sleep, dyspnea, and spinal malformations, vision problems that can lead to blindness, headaches, hydrocephalus, aorta problems (Identifiable by CT) and even dermatological problems.

Accurate and early diagnosis allows better management of future patient issues such as mental retarder, airway obstructions and a constant decrease in visual ability. With the right treatments, people can be as productive and active as most of society [57]. Numerous specialists such as pediatricians, maxillofacialists, neurosurgeons, otolaryngologists and an ophthalmologist are involved to manage people with this pathology. If the abnormalities of the cranial vault are corrected the patient will have normal cognitive functions, sight and hearing. Other specialists may be required in relation to the malformations. There are some people with SC, whose pathological course does not involve surgery; in this case the team of doctors will follow the person to prevent the development of complications.

Analysis of craniosynostosis-related FGFR receptor inhibitors is currently under way, but none of them have been tested in humans [57].

\section{CONCLUSIONS}

In conclusion, there is a known correlation between certain systemic pathologies and the oral cavity, which can also be highlighted by imaging in the dental field.

It is important for dentists to know these diseases, rare but present, in order to help the patient's diagnostic path, sometimes managing to anticipate a pathology not yet known.

The anamnestic assessment associated with simple radiological examinations, in its competence, promotes a holistic view of the profession, does not simply limit it to the inspection of the oral cavity aimed at the dental elements but integrates it with the overall view often improving the prognosis and quality of life of the patient.

Correlations between oral and systemic alterations are often so correlated that they put the entire dental team in a privileged situation in early diagnosis. Multidisciplinary interaction with other specialists is crucial for patient management.

\section{REFERENCES}

1. Adisen MZ, Okkesim A, Misirlioglu M. The importance of early diagnosis of gardner's syndrome in dental examination. Nigerian journal of clinical practice. 2018;21(1):114-6.

2. Woo VL, Abdelsayed R. Oral manifestations of internal malignancy and paraneoplastic syndromes. Dental Clinics of North America. 2008 Jan 1;52(1):203-30.

3. Milicevic M. Lésions mixtes des mâchoires, radioopaques et radiotransparentes. Revue Médicale de Liège. 2017;72(3):151-5.

4. Almazrooa S, Binmadi NO, Khalifa HM, Jadu FM, Jan AM, Meisha DE. The agreement rate between radiographic interpretation and histopathologic diagnosis of jaw lesions. Radiology Research and Practice. 2019 Mar 4;2019.

5. Ficarra G. Manuale di patologia e medicina orale. McGraw-Hill libri Italia; 1998.

6. Patankar SR, Khetan P, Choudhari SK, Suryavanshi H. Dentinogenic ghost cell tumor: A case report. World journal of clinical oncology. 2019 Apr 24;10(4):192.

7. Bafna SS, Joy T, Tupkari JV, Landge JS. "Dentinogenic ghost cell tumor." J Oral Maxillofac Pathol 2016; 20: 163 
8. Agrawal Y, Naidu GS, Makkad RS, Nagi R, Jain S, Gadewar DR, Kataria R. Dentinogenic ghost cell tumor-a rare case report with review of literature. Quantitative Imaging in Medicine and Surgery. 2017 Oct;7(5):598.

9. Barnes L, Eveson JW, Reichart P, Sidransky D. "World Health Organization Classification of Tumours. Pathology and Genetics of Head and Neck Tumours.” Lyon: IARC Press. 2005:314.

10. Juneja M, George J. Dentinogenic ghost cell tumor: a case report and review of the literature. Oral Surgery, Oral Medicine, Oral Pathology, Oral Radiology, and Endodontology. 2009 May 1;107(5):e17-22.

11. Praetorius F. "Dentinogenic ghost cell tumour." In: Barnes L. editor. Surgical Pathology of the Head and Neck, Third Edition. CRC Press; 2009:1272

12. Garcia BG, Ruiz Masera JJ, Zafra Camacho FM, Gutierrez CC. "Intraosseous dentinogenic ghost cell tumor: Case report and treatment review." Rev Esp Cir Oral Maxilofac. 2015;37:243-6.

13. Bussari S, Thakur SM, Koshy AV, Shah AA. "Dentinogenic ghost cell tumor - A case report and review of literature." J Oral Maxillofac Pathol. 2019;23(Suppl 1):66-68.

14. Gorlin RJ, Pindborg JJ, Clausen FP, Vickers RA. The calcifying odontogenic cyst- a possible analogue of cutaneous calcifying epithelioma of Malherbe. Oral Surg Oral Med Oral Pathol. 1962; 15: 1235-43.

15. Singhaniya SB, Barpande SR, Bhavthankar JD. "Dentinogenic ghost cell tumor." J Oral Maxillofac Pathol. 2009; 13: 97-100

16. Kim SA, Ahn SG, Kim SG, Park JC, Lee SH, Kim J. "Investigation of the beta-catenin gene in a case of dentinogenic ghost cell tumor." Oral Surg Oral Med Oral Pathol Oral Radiol Endod. 2007; 103:97-101.

17. Patil NN, Nayyar AS, Wadhwan V. "Adenomatoid odontogenic tumor: A series of four clinicopathological variants" Int J Case Rep Imag. 2014; (1): 1,7 .

18. Cicconetti A, Tallarico M, Bartoli A, Ripari A, Maggiani F. "Calcifying epithelial odontogenic (Pindborg) tumor. A clinical case." Minerva Stomatol. 2004; 53:379-87.

19. Rappaport MJ, Showell DL, Edenfield WJ. "Metastatic Ghost Cell Odontogenic Carcinoma: Description of a Case and Search for Actionable Targets." Rare Tumors. 2015;7(3):5813.

20. Campolongo MG, Cabras M, Bava L, Arduino PG, Carbone M. "Paget's disease of jaw bones as primary manifestation: A case report of a proper diagnosis made by general dentist." Gerodontology. 2018;35:147-150.

21. Karunakaran K, Murugesan P, Rajeshwar G, Babu S. "Paget's disease of the mandible." J Oral Maxillofac Pathol. 2012;16(1):107-109.

22. Jarrah Ali Al-Tubaikh, Maximilian F. Reiser, "Congenital Diseases and Syndromes, An
Illustrated Radiological Guide," Springer-Verlag Berlin Heidelberg; 2009.

23. Watkinson JC, Clarke RW, editors. Scott-Brown's Otorhinolaryngology and Head and Neck Surgery: Volume 2: Paediatrics, The Ear, and Skull Base Surgery. CRC Press; 2018 Jun 12.

24. Amaral MA, Falcão AS, Miranda MS, Félix VB, de Melo Alves Jr S, da Silva Kataoka MS. Dental implants in a patient with Paget's disease: a case report. Revista Odonto Ciência. 2014 Oct $1 ; 29(4): 125-8$.

25. Kumar V, Abbas AK, Aster JC. "Robbins e CotranLe basi patologiche delle malattie", $9^{\text {a }}$ edizione, vol. 2,1154, Edra

26. You M, Tang B, Wang ZJ, Wang KL, Wang H, "Radiological manifestations of renal osteodystrophy in the orofacial region: a case report and literature review", Oral Radiol. 2018; 34(3):262-266.

27. Proctor R, Kumar N, Stein A, Moles D, Porter S. "Oral and dental aspects of chronic renal failure." J Dent Res. 2005; 84:199-208.

28. Pontes FS, Lopes MA, de Souza LL, da Mata Rezende DD, Santos-Silva AR, Jorge Jr J, da Silva WG, Pires FR, Rocha AC, de Campos WG, Caldato MC. Oral and maxillofacial manifestations of chronic kidney disease-mineral and bone disorder: a multicenter retrospective study. Oral Surgery, Oral Medicine, Oral Pathology and Oral Radiology. 2018 Jan 1;125(1):31-43.

29. Parthiban J, Aarthi Nisha V, Asokan GS, Prakash CA, Varadharaja MM. Oral manifestations in a renal osteodystrophy patient-a case report with review of literature. Journal of clinical and diagnostic research: JCDR. 2014 Aug;8(8):ZD28.

30. Gu GL, Wang SL, Wei XM, Bai L. Diagnosis and treatment of Gardner syndrome with gastric polyposis: a case report and review of the literature. World Journal of Gastroenterology: WJG. 2008 Apr 7;14(13):2121.

31. Fotiadis C, Tsekouras DK, Antonakis $\mathrm{P}$, Sfiniadakis J, Genetzakis M, Zografos GC "Gardner's syndrome: A case report and review of the literature" World J Gastroenterol. 2005; 11(34): 5408-5411

32. Dinarvand P, Davaro EP, Doan JV, Ising ME, Evans NR, Phillips NJ, Lai J, Guzman MA. Familial adenomatous polyposis syndrome: an update and review of extraintestinal manifestations. Archives of pathology \& laboratory medicine. 2019 Nov;143(11):1382-98.

33. Ida M, Nakamura T, Utsunomiya J. Osteomatous changes and tooth abnormalities found in the jaws of patients with adenomatosis coli. Oral Surgery, Oral Medicine, Oral Pathology. 1981 Jul 1;52(1):211.

34. Baldino ME, Koth VS, Silva DN, Figueiredo MA, Salum FG, Cherubini K. Gardner syndrome with maxillofacial manifestation: A case report. Special Care in Dentistry. 2019 Jan;39(1):65-71. 
Viganò Luca et al., Sch J Dent Sci, Nov, 2020; 7(11): 183-195

35. Smud D, Augustin G, Kekez T, Kinda E, Majerovic M, Jelincic Z. Gardner's syndrome: genetic testing and colonoscopy are indicated in adolescents and young adults with cranial osteomas: a case report. World journal of gastroenterology: WJG. 2007 Jul 28;13(28):3900.

36. Koh KJ, Park HN, Kim KA. Gardner syndrome associated with multiple osteomas, intestinal polyposis, and epidermoid cysts. Imaging science in dentistry. 2016 Dec 1;46(4):267-72.

37. Yu F, Cai W, Jiang B, Xu L, Liu S, Zhao S. A novel mutation of adenomatous polyposis coli (APC) gene results in the formation of supernumerary teeth. Journal of Cellular and Molecular Medicine. 2018 Jan;22(1):152-62.

38. Argyris PP, Koutlas IG. "Orthokeratinized Odontogenic Cyst with an Associated Keratocystic Odontogenic Tumor Component and Ghost Cell Keratinization and Calcifications in a Patient with Gardner Syndrome" Head and Neck Pathol. 2017; 11:240-248

39. Salti L, Rasse M, Al-ouf K "Maxillofacial Radiographic study of Gardner's syndrome presenting with odontogenic myxoma: A rare case report" Stomatologija, Baltic Dental and Maxillofacial Journal. 2018; 20:59

40. Stimolo M, De Riu G, Meloni SM, Piombino P, Tullio A. Maxillary odontogenic myxoma: case report and literature review. DENTAL CADMOS. 2015 Jan 1;83(1):46-51.

41. Trinh CT, Nguyen $\mathrm{CH}$, Chansomphou V, Chansomphou V, Tran TT. Overview of epidermoid cyst. European journal of radiology open. 2019 Jan 1;6:291-301.

42. Yu D, Benjamin NG, Zhu H, Liu J, Lin Y. Bone and dental abnormalities as first signs of familial Gardner's syndrome in a Chinese family: a literature review and a case report. médecine/sciences. 2018 Oct 1;34:20-5.

43. Lyons LA, Lewis RA, Strong LC, Zuckerbrod S, Ferrell RE. A genetic study of Gardner syndrome and congenital hypertrophy of the retinal pigment epithelium. American journal of human genetics. 1988 Feb;42(2):290.

44. Davies DR, Armstrong JG, Thakker N, Horner K, Guy SP, Clancy T, Sloan P, Blair V, Dodd C, Warnes TW, Harris R. Severe Gardner syndrome in families with mutations restricted to a specific region of the APC gene. American journal of human genetics. 1995 Nov;57(5):1151.

45. Hegde K, Kashyap RR, Nair G, Nair PP. Familial ectodermal dysplasia: a peers' agony. Case Reports. 2013 Jul 23;2013:bcr2013200175.
46. Dellavia C, Sforza C, Malerba A, Strohmenger L, Ferrario VF. Palatal size and shape in 6-year olds affected by hypohidrotic ectodermal dysplasia. The Angle Orthodontist. 2006 Nov;76(6):978-83.

47. Rosa RR, Janeiro MM, Camargo SE, Porto CD, Kreich EM, Henriques JC. Radiographic study of patients with ectodermal dysplasia and partial. Indian Journal of Dental Research. 2012 Nov $1 ; 23(6): 801$.

48. García de Marcos JA, Dean Ferrer A, Alamillos Granados F, Ruiz Masera JJ, Barrios Sánchez G, Romero Ortiz AI, Calderón Bohórquez JM, Valenzuela Salas B. Langerhans cell histiocytosis in the maxillofacial area in adults: Report of three cases. Medicina Oral, Patología Oral y Cirugía Bucal (Internet). 2007 Mar;12(2):145-50.

49. Kim JE, Yi WJ, Heo MS, Lee SS, Choi SC, Huh KH. Langerhans cell histiocytosis of the jaw, a mimicker of osteomyelitis on CT and MR images: a retrospective analysis. Medicine. 2019 Jul;98(27).

50. Schepman KP, Raddent BG, Van der Waal I. Langerhans' cell histiocytosis of the jaw bones. Report of 11 cases. Australian dental journal. 1998 Aug;43(4):238-41.

51. Conrady CD, Patel BC. Crouzon Syndrome. InStatPearls [Internet] 2019 Apr 10. StatPearls Publishing.

52. Reardon W, Winter RM, Rutland P, Pulleyn LJ, Jones BM, Malcolm S. Mutations in the fibroblast growth factor receptor 2 gene cause Crouzon syndrome. Nat. Genet. 1994 Sep;8(1):98-103

53. Snyder-Warwick AK, Perlyn CA, Pan J, Yu K, Zhang L, Ornitz DM. Analysis of a gain-offunction FGFR2 Crouzon mutation provides evidence of loss of function activity in the etiology of cleft palate. Proc. Natl. Acad. Sci. U.S.A. 2010 Feb 09; 107(6):2515-20.

54. Crouzon LE. Dysostose cranio-faciale héréditaire. Bulletin de la Société des Médecins des Hôpitaux de Paris. 1912;33:545-555.

55. Santos AP, Ammari MM, Moliterno LF, Júnior JC. First report of bilateral supernumerary teeth associated with both primary and permanent maxillary canines. J Oral Sci. 2009;51:145-50

56. Raviprakash Sasankoti Mohan, Naveen Shanker Vemanna, Sankalp Verma, and Neha Agarwal. Crouzon Syndrome: Clinico-Radiological Illustration of a Case. Uttar Pradesh, India; 2012

57. Hlongwa P. Early orthodontic management of Crouzon syndrome: A case report. J Maxillofac Oral Surg. 2009;8:74-6. 